\title{
Jacobsen Syndrome with White Matter Changes
}

\author{
Sejin Ahn, MD, In-Sang Jeon, MD, Dong Woo Son, MD, Kyung Jin Ahn, MD, Kyung In Lim, MD, \\ Hyo Jeong Kim, MD \\ Department of Pediatrics, Gachon University Gil Medical Center, Gachon University College of Medicine, Incheon, Korea
}

Received: March 15, 2021

Revised: April 3, 2021

Accepted: April 7, 2021

Corresponding author:

Hyo Jeong Kim, MD

Department of Pediatrics, Gachon University Gil Medical Center, Gachon University College of Medicine, 21 Namdong-daero 774beon-gil, Namdong-gu, Incheon 21565, Korea

Tel: +82-32-460-3224

Fax: +82-32-460-2362

E-mail: greatelena@naver.com
Jacobsen syndrome is a rare genetic disorder caused by partial deletions in the long arm of chromosome 11 . The deletion size ranges from 7 to $20 \mathrm{Mb}$, with a breakpoint at 11q23.3, in $70 \%$ to $80 \%$ of cases. Affected individuals can express a wide variety of phenotypes, including delayed physical growth and psychomotor development, dysmorphic features, congenital heart malformations, and thrombocytopenia [1,2]. Few reports have described white matter abnormalities in Jacobsen syndrome. Herein, we describe a case of a boy diagnosed with Jacobsen syndrome with white matter abnormalities on brain magnetic resonance imaging (MRI). This study was reviewed and approved by the Institutional Review Board of the Gachon University Gil Hospital (GFIRB 2020-458). Due to its retrospective nature, the study was exempt from requiring informed consent from the participants.

A 6-day-old neonate was brought to our institution because of jaundice. He was born at 37 weeks of gestation without perinatal asphyxia. His birth weight was 2,980 g (22nd percentile), height was $46 \mathrm{~cm}$ (2nd percentile), and head circumference was $33 \mathrm{~cm}$ (13th percentile). Physical examination revealed a cephalohematoma. Laboratory tests showed hyperbilirubinemia, hypothyroidism, neutropenia, and thrombocytopenia. The total bilirubin level was $25.5 \mathrm{mg} /$ $\mathrm{dL}$. The white blood cell count was $2,070 / \mu \mathrm{L}$, hemoglobin was $10.7 \mathrm{~g} / \mathrm{dL}$, and platelet count was $9,000 / \mu \mathrm{L}$. During 1 month of hospitalization, thrombocytopenia persisted despite treatment with intravenous immunoglobulin, steroids, and transfusion. After discharge, the patient's blood cell counts were regularly monitored through follow-up. A chromosomal study was not performed at the time.

He visited our neurology clinic because of developmental delay at 2 years of age. As he grew up, his height remained below the 3 rd percentile. At 2 years of age, his height was $81 \mathrm{~cm}$ (2nd percentile), weight was $11 \mathrm{~kg}$ (19th percentile), and head circumference was $47 \mathrm{~cm}$ (18th percentile). He had a dysmorphic face, including a high prominent forehead, down-slanting palpebral fissures, short nose, flat nasal bridge, and thin upper lip. He could speak only the word "mama" unclearly. He started walking at 19 months of age. The Bayley scale was used to assess his degree of development at 25 months of age, and showed that his cognition and motor skills were at the 1st percentile, whereas language was below the 0.1 percentile.

An assessment of language development via the Sequenced Language Scale for Infants, conducted at 25 months of age, showed that the patient's language development was equivalent to that of a 6-month-old child.

Brain MRI performed at 24 months of age showed focal and patchy $\mathrm{T} 2$ high-signal-intensity lesions in the bilateral frontal and parietal sub-

Copyright (C) 2021 Korean Child Neurology Society

This is an Open Access article distributed under the terms of the Creative Commons Attribution Non-Commercial License (http://creativecommons.org/licenses/by-nc/4.0/) which permits unrestricted non-commercial use, distribution, and reproduction in any medium, provided the original work is properly cited. 


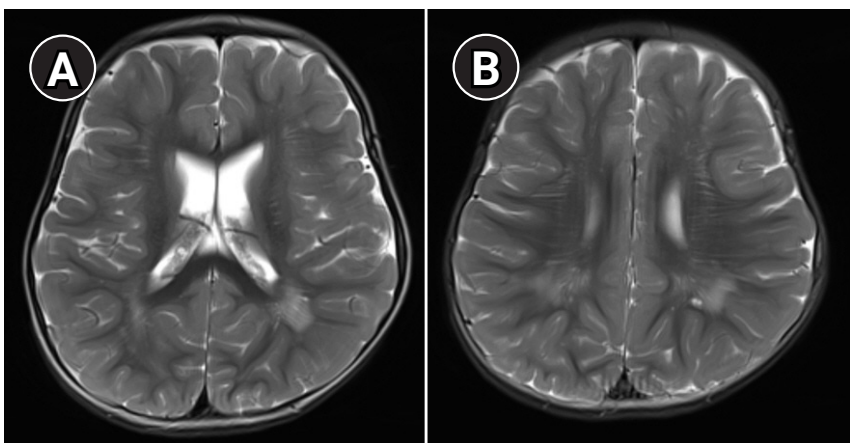

Fig. 1. (A, B) T2-weighted brain magnetic resonance imaging showed focal and patchy high-signal-intensity lesions in the bilateral frontal and parietal subcortical white matter.

cortical white matter (Fig. 1). Electroencephalography revealed no abnormalities. Echocardiography revealed a small atrial septal defect (secundum type). Abdominal ultrasonography showed no visceral malformations including the liver, kidney, and intestines. Ophthalmologic evaluations and hearing tests were normal.

Chromosomal microarray analysis confirmed a $12.6-\mathrm{Mb}$ deletion in 11q24.1q25, which resulted in the diagnosis of Jacobsen syndrome. Therefore, we could conclude that Jacobsen syndrome was the reason for the patient's developmental delay, intellectual disability, and physical growth retardation due to the deletions in chromosome 11.

Currently, at the age of 4 years and 10 months, his height is 102 $\mathrm{cm}$ (6th percentile), body weight is $19.4 \mathrm{~kg}$ (60th percentile), and head circumference is $50.1 \mathrm{~cm}$ (25th percentile). He can speak about five words, including "mama" and "papa." According to the most recent laboratory tests, his white blood cell count was 4,500/ $\mu \mathrm{L}$, hemoglobin was $11.9 \mathrm{~g} / \mathrm{dL}$, and platelet count was 119,000 / $\mu \mathrm{L}$.

Jacobsen syndrome is caused by partial deletion of genes on the long arm of chromosome 11 [1]. Its prevalence has been estimated at 1/100,000 births, with a female-to-male ratio of 2:1 [3]. Common clinical features of Jacobsen syndrome include physical growth retardation, psychomotor retardation, facial dysmorphism, and thrombocytopenia or pancytopenia [3]. Some patients have visceral malformations involving the heart, kidney, gastrointestinal tract, genitalia, or central nervous system. Ocular, hearing, immunological, and hormonal problems may also occur [1,2]. During the neonatal period, most patients are hospitalized for prolonged periods due to feeding difficulties, cardiac problems, or bleeding diathesis [3]. Our patient had a prolonged hospitalization due to thrombocytopenia. He had growth retardation, psychomotor retardation, facial dysmorphism, thrombocytopenia, neutropenia, and white matter changes on brain MRI. The characteristic clinical aspects and chromosomal analysis led to the diagnosis.
Approximately $65 \%$ of patients with Jacobsen syndrome have a structural abnormality of the brain such as ventriculomegaly, cerebral atrophy, agenesis of the corpus callosum, or pachygyria [3]. Cerebral white matter abnormalities in Jacobsen syndrome have also been reported, but detailed data regarding the pathophysiology, radiological characteristics, and clinical course are lacking.

White matter abnormalities in Jacobsen syndrome are thought to be associated with the deletion of HEPACAM located on 11q24.2, which causes white matter water retention and intra-myelinic edema [4]. HEPACAM is the causative gene in megalencephalic leukoencephalopathy with subcortical cysts (MLC) type $2 \mathrm{~A}$ and $2 \mathrm{~B}$. MLC is characterized by chronic white matter edema, macrocephaly, and subcortical cysts [5]. Although Jacobsen syndrome does not present other features of MLC except white matter edema, HEPACAM haploinsufficiency in Jacobsen syndrome might have a potential role in the pathogenesis of white matter changes. Fujino et al. [5] documented serial white matter changes from the neonatal period to young childhood. Brain MRI taken before the age of 2 years shows more extensive or diffuse white matter changes. In contrast, after 2 years of age, white matter changes are mostly mild or partial [5]. White matter abnormalities and progression of myelination improve over time.

In conclusion, it should be noted that when clinical features suggest the presence of Jacobsen syndrome, the patient may also have nonspecific white matter abnormalities on brain MRI. Awareness of the presentation of Jacobsen syndrome on brain MRI is important to prevent unnecessary evaluation.

\section{Conflicts of interest}

No potential conflict of interest relevant to this article was reported.

\section{ORCID}

Sejin Ahn, https://orcid.org/0000-0002-5161-2467

Hyo Jeong Kim, https://orcid.org/0000-0003-2191-5341

\section{Author contribution}

Conceptualization: HJK. Data curation: SA, ISJ, DWS, KJA, KIL, and HJK. Formal analysis: SA and HJK. Methodology: SA, ISJ, DWS, KJA, KIL, and HJK. Project administration: HJK. Visualization: SA and HJK. Writing-original draft: SA. Writing-review \& editing: HJK. 


\section{References}

1. Jacobsen P, Hauge M, Henningsen K, Hobolth N, Mikkelsen M, Philip J. An $(11 ; 21)$ translocation in four generations with chromosome 11 abnormalities in the offspring. A clinical, cytogenetical, and gene marker study. Hum Hered 1973;23:568-85.

2. Penny LA, Dell'Aquila M, Jones MC, Bergoffen J, Cunniff C, Fryns JP, et al. Clinical and molecular characterization of patients with distal 11q deletions. Am J Hum Genet 1995;56:67683.
3. Mattina T, Perrotta CS, Grossfeld P. Jacobsen syndrome. Orphanet J Rare Dis 2009;4:9.

4. Yamamoto T, Shimada S, Shimojima K, Sangu N, Ninomiya S, Kubota M. Leukoencephalopathy associated with 11q24 deletion involving the gene encoding hepatic and glial cell adhesion molecule in two patients. Eur J Med Genet 2015;58:492-6.

5. Fujino S, Yoshihashi H, Takeda R, Ihara S, Miyama S. White matter abnormality in Jacobsen syndrome assessed by serial MRI. Brain Dev 2020;42:621-5. 
